Para citar este artículo: López-Sánchez, U., Onofre-Rodríguez, D. J., Torres-Obregon, R., Benavides-Torres, R. A., \& Garza-Elizondo, M. E. (2021). Hipermasculinidad y uso de condón en hombres que tienen sexo con hombres y mujeres. Health and Addictions / Salud y Drogas, 21(1), 63-75. doi:10.21134/haaj.v21i1.510

\title{
HIPERMASCULINIDAD Y USO DE CONDÓN EN HOMBRES QUE TIENEN SEXO CON HOMBRES Y MUJERES
}

\author{
HYPERMASCULINITY AND CONDOM USE IN MEN WHO HAVE SEX WITH MEN AND \\ WOMEN \\ Ulises López-Sánchez, Dora J. Onofre-Rodríguez, Reyna Torres-Obregon, Raquel A. \\ Benavides-Torres y María E. Garza-Elizondo
}

\author{
Universidad Autónoma de Nuevo León (México)
}

\begin{abstract}
Los hombres que tienen sexo con hombres y mujeres (HSHM) conforman un subgrupo de hombres que tienen sexo con hombres, son una población clave con mayor riesgo de exposición al virus de inmunodeficiencia humana. Al respecto, un método eficaz de prevención es el uso de condón, sin embargo, esta población se ve afectada por factores contextuales y sociales como la hipermasculinidad, la cual podría estar limitando la conducta sexual segura. Objetivo. Determinar la influencia de la hipermasculinidad sobre el uso de condón en hombres que tienen sexo con hombres y mujeres. Metodología. Estudio transversal correlacional en 106 HSHM. Se utilizó el muestreo virtual online, dos instrumentos y una cédula de datos sociodemográficos y de historia sexual. Resultados. Se encontró que la hipermasculinidad influye de manera negativa sobre el uso del condón ( $B=-.318, p=.001)$. Conclusiones. Las ideas hipermasculinas, están asociadas a menor uso de condón en los HSHM, los hallazgos sugieren que existe la necesidad de diseñar intervenciones específicas en el contexto de este subgrupo de hombres enfocadas a aumentar el uso de condón y contemplen los componentes de la ideología masculina.
\end{abstract}

Palabras clave: hombres que tienen sexo con hombres y mujeres, hipermasculinidad, uso de condón

Men who have sex with men and women (MSMW) make up a subgroup of men who have sex with men, they are a key population with a higher risk of exposure to the human immunodeficiency virus. In this regard, an effective method of prevention is the condom use, however, this population is affected by contextual and social factors such as hypermasculinity, which could be limiting safe sexual behavior. Objective. Determine the influence of hypermasculinity on condom use in men who have sex with men and women. Methodology. A descriptive-correlational design was used in $106 \mathrm{MSMW}$. Virtual snowball sampling, two instruments and a sociodemographic data and sexual history card were used. Results. It was found that hypermasculinity has a negative influence on condom use $(B=-.318, p=.001)$. Conclusions. Hypermasculine ideas are associated with less condom use in MSMW, the findings suggest that there is a need to design specific interventions in the context of this subgroup of men focused on increasing condom use and considering the components of masculine ideology.

Keywords: men who have sex with men and women, hypermasculinity, condom use.

Ulises López-Sánchez, Facultad de Enfermería, Universidad Autónoma de Nuevo León, México, https://orcid.org/0000-0003-13393141.

Dora Julia Onofre-Rodríguez, Facultad de Enfermería y Centro de Investigación y Desarrollo en Ciencias de la Salud, Universidad Autónoma de Nuevo León, México, correspondencia donofre64@yahoo.com.mx, https://orcid.org/0000-0003-1214-9761.

Reyna Torres-Obregon, Facultad de Enfermería, Universidad Autónoma de Coahuila, Unidad Saltillo, https://orcid.org/0000-00033546-6970.

Raquel A. Benavides-Torres, Facultad de Enfermería y Centro de Investigación y Desarrollo en Ciencias de la Salud, Universidad Autónoma de Nuevo León, México, https://orcid.org/0000-0001-5113-4250

María Eugenia Garza-Elizondo, Escuela de Enfermería del Instituto Mexicano del Seguro Social (IMSS), Delegación Nuevo León, https://orcid.org/0000-0001-9181-7136 
A nivel mundial se estima que 36.9 millones de personas viven con el virus de inmunodeficiencia humana (VIH), hay 1.8 millones de nuevos casos y un millón de personas fallecieron por causas relacionadas con este virus (Programa Conjunto de las Naciones Unidas sobre el VIH y el Sida [ONUSIDA], 2017]). Lo que respecta a México, 301 mil 182 personas con infección por VIH se encuentran en el registro de vigilancia epidemiológica, en el año 2018 se notificaron 10701 casos nuevos del VIH y 6287 casos nuevos de sida, concentrándose la mayor parte en el grupo entre 25 y 44 años. Por sus características, el VIH en México, se considera una epidemia concentrada, en la cual las poblaciones más afectadas son: hombres que tienen sexo con otros hombres (HSH), hombres trabajadores sexuales (HTS), mujeres trans (MT), personas que se inyectan drogas (PID) y reclusos (Centro Nacional para la Prevención y Control del VIH y el Sida [CENSIDA], 2019).

Entre los HSH, los hombres que tienen sexo con hombres y mujeres (HSHM), tiene más probabilidad de informar comportamientos que aumentan el riesgo del VIH en comparación con los HSH con parejas masculinas únicamente (Centros para el Control y la Prevención de Enfermedades, [CDC] 2019; Shadaker, Magee, Paz, \& Hoots, 2017; Xu, Zheng, Liu, \& Zheng, 2016). En México, la prevalencia del VIH en HSHM de entre 20 y 49 años es del $4.4 \%$, se estima que la comunidad de HSHM es de 603406 hombres, de los cuales, con la prevalencia estimada, habría 26550 casos con VIH positivos (Gutiérrez et al., 2014).

La alta prevalencia de la infección por el VIH y el aumento de la probabilidad de comportamiento bisexual entre los HSH hace imperativo se comprenda el riesgo del VIH y las necesidades de prevención de los HSHM. La literatura muestra que a diferencia de los hombres con prácticas sexuales heterosexuales, los HSHM tienen menos probabilidad de participar en programas o actividades de prevención del VIH (Ellen et al., 2015; Friedman et al., 2014), más probabilidad que practiquen el sexo anal sin condón con parejas ocasionales (Grov, Golub, Mustanski, \& Parsons, 2010), de buscar intencionalmente el sexo anal sin condón (Scanavino et al., 2018) y por ende, más posibilidad de adquirir el VIH (McCree et al., 2017).

De acuerdo con lo anterior, ONUSIDA, (2017), propone poner fin a la epidemia para el año 2030, esta acción acelerada busca reducir en un 75\% las nuevas infecciones en los grupos de población clave, entre ellos los HSHM, a través de la promoción de prácticas sexuales seguras (Maulsby, Sifakis, German, Flynn, \& Holtgrave, 2013; Shadaker, Magee, Paz, \& Hoots, 2017; Xu, Zheng, Liu, \& Zheng, 2016). Así mismo, la literatura refiere que existen factores de relevancia que podrían repercutir en las prácticas sexuales seguras, como los factores sociales, considerados como los de mayor importancia en la incidencia del VIH en este grupo clave, por ejemplo, la ideología de la masculinidad, la cual puede desempeñar un papel único en las conductas de riesgo del VIH (Zeglin \& Stein, 2015).

La ideología de la masculinidad se refiere la adaptación interiorizada por un individuo de las normas del rol masculino, que se diferencia de las normas culturales en formas que minimizan el conflicto del rol de género de ese individuo (O'Neil et al., 1986). Es decir, es una construcción social de una cuestión de poder que está asociada al quehacer del varón y a su capacidad para ejercer el dominio y el control, que conduce a una posición hegemónica sobre las mujeres y los homosexuales y forma parte de cualquier organización social. A menudo se construye en términos de posturas o ideologías hipermasculinas, estas se caracterizan por la exageración de lo que se considera como "lo masculino", tanto en el cuerpo como en el comportamiento con tendencia a la rudeza, violencia, falta de afectividad y agresividad, las cuales no se establecen per 
se, sino como adopción de ciertos comportamientos asociados al dominio y al poder (Viveros, 2001). Estas ideologías hipermasculinas se han incluido en investigaciones relacionadas con la divulgación de la orientación sexual, el estado serológico al VIH, con la educación y con la manera de seleccionar a las parejas sexuales (Bianchi et al., 2017; Clark et al., 2013; Knox, Reddy, Kaighobadi, Nel \& Sandfort, 2013). Investigación previa ha sugerido que la ideología hipermasculina se ha asociado con tasas más altas de múltiples parejas sexuales y una aversión a utilizar condón (Oparanozie, Sales, Di Clemente, \& Braxton 2012; Rhodes et al., 2011).

Se ha encontrado que los hombres con ideologías masculinas más tradicionales es más probable que participen en conductas sexuales de riesgo, como sexo oral, vaginal y anal sin protección (Santana, Raj, Decker, La Marche \& Silverman, 2006) y reportan frecuencias más bajas de los comportamientos que promueven la salud en general (Mahalik, Burns \& Syzdek, 2007). Con respecto a ello, una de las formas más efectivas de prevenir la transmisión del VIH, es mediante conducta sexual segura, sin embargo, a pesar de que han pasado más de tres décadas de los primeros casos del VIH, aún no se ha logrado identificar con claridad por qué las personas no adoptan esta medida preventiva. El sexo seguro es un término integral que refleja que es posible elegir y adoptar conductas para reducir o minimizar el riesgo de infección por el VIH, entre estas están hacer un uso correcto y sistemático del condón (CDC, 2019; da Fonte et al., 2017). Consecuentemente, el uso de condón representa un factor protector para prevenir la infección por VIH, se estima que el uso constante de este puede disminuir en un $80 \%$ la incidencia del VIH (Martínez-Torres, Parada-Capacho, \& Castro-Duarte, 2014).

Dentro de una cultura particular, la ideología de la masculinidad aunado a los correlatos de sexo seguro necesita una mayor exploración, especialmente en culturas y subculturas en las que ha habido poca o ninguna investigación cuantitativa, de ahí la necesidad de examinar la influencia de la hipermasculinidad en el uso de condón que pueda disminuir el riesgo del VIH en el grupo de los HSHM.

\section{Método}

Estudio con diseño transversal-correlacional.

\section{Participantes}

La población estuvo conformada por HSHM del noreste de México. Se utilizó un muestreo virtual online, ideal para acceder a poblaciones de baja incidencia o de difícil acceso (Baltar \& Gorjup, 2012). El tamaño de la muestra se estimó mediante el paquete estadístico n'Query Advisor, con un nivel de significancia de .05 , un Coeficiente de Determinación $R^{2}=.10$ y una potencia de prueba del $80 \%$. El total de la muestra fue de 106 HSHM. Se seleccionaron participantes mayores de 18 años, haber tenido relaciones sexuales anales, vaginales $u$ orales en los últimos tres meses con al menos un hombre y una mujer. Los criterios de eliminación fueron ser VIH positivos y reportar trabajo sexual como ocupación.

\section{Instrumentos}

Datos sociodemográficos y de historia sexual. Para caracterizar la muestra del estudio se indagó edad, estado civil, último grado de estudios, orientación sexual, edad en la que tuvo la primera relación sexual, número de parejas sexuales en el último año, uso de condón en la primera y última relación sexual, con quién tuvo su última relación sexual, ha pagado o recibido 
dinero por sexo, conocimiento del estado serológico al VIH y frecuencia de pruebas de detección del $\mathrm{VIH}$.

Hipermasculinidad. Se utilizó la escala Multicultural de Ideología Masculina (Multicultural Masculinity Ideology Scale, MMIS; Doss \& Hopkins, 1998), con la subescala de ideología hipermasculina, desarrollada y validada en población americana, traducida al español para fines del presente estudio con el método de "Back Translation" (Burns \& Grove, 2012). Está conformada por 13 ítems, con cuatro opciones de respuesta tipo Likert que van de $1=$ totalmente en desacuerdo, hasta 4= totalmente de acuerdo. Las puntuaciones oscilan entre 13 y 52 . Una mayor puntuación en la subescala indica mayor ideología masculina. La escala se ha utilizado en HSHM, presentando Alfas de Cronbach aceptables de .83 y .87 (LaPollo et al., 2014).

Uso de condón. Se empleó la subescala Uso del condón del Cuestionario de Comportamiento Sexual Seguro (Dilorio, Parsons, Lehr, Adame, \& Carlone, 1992), consta de 8 ítems con respuestas de tipo Likert que van desde "nunca" (1) hasta "siempre" (4). Las puntuaciones oscilan entre 8 y 32, los puntajes más altos indican mayor uso de condón. La escala ha sido aplicada en diferentes poblaciones y países, incluyendo al grupo de HSH y HSHM en México, donde se han obtenido Alfas de Cronbach de .91 (Dilorio et al., 1992; Jiménez, 2018; Valdez, 2015).

\section{Procedimiento}

El estudio fue autorizado por el Comité de Investigación (No.13Cl19039006) y por el Comité de Ética en Investigación (No.19CEI00420180614) de la Facultad de Enfermería de la Universidad Autónoma de Nuevo León. Con fundamento en lo establecido en el Reglamento de la Ley General de Salud en Materia de Investigación para la Salud (Secretaría de Salud [SS], 1987). Para todos los casos se obtuvo el consentimiento informado online. De acuerdo con la metodología del muestreo virtual online, se utilizó una encuesta auto aplicable a través del software QuestionPro para encuestas online, el cual incluía, consentimiento informado, datos sociodemográficos y de historia sexual, y los instrumentos de medición antes mencionados. Para el reclutamiento, se difundió un enlace y una invitación del estudio en páginas de asociaciones civiles de la comunidad de Lesbianas, Gays, Bisexuales, Trans y más (LGBT+), grupos de Facebook relacionados a la diversidad sexual, y en aplicaciones móviles de citas como Tinder y Grindr, aclarando que se utilizaron estas asociaciones, ya que el grupo de HSHM se considera una muestra oculta o de difícil acceso.

Quienes decidieron participar en el estudio, dieron clic en el enlace el cual los redireccionó al inicio de la encuesta antecedida por el consentimiento informado, en donde se explicó el objetivo del estudio y se proporcionaron datos de contacto de los investigadores, esto último con el fin disipar dudas respecto al estudio. Después, los participantes potenciales tuvieron la oportunidad de decidir iniciar o declinar la encuesta. QuestionPro notificó al investigador a través de correo electrónico por cada encuesta contestada y el tiempo aproximado del llenado, el cual fue de seis minutos en promedio. Después de la notificación, el investigador procedía a seleccionar de las encuestas contestadas, aquellos que cumplieran con los criterios de inclusión para posteriormente exportar la base de datos al paquete estadístico Statistical Package for Social Sciences (SPSS) versión 22. Los participantes fueron reclutados entre los meses de mayo y agosto del 2019. 


\section{Análisis de datos}

Se utilizó estadística descriptiva para datos sociodemográficos y de historia sexual. Se aplicó correlación de Spearman y un modelo de regresión lineal simple.

\section{Resultados}

La edad de los participantes osciló entre 18 y 61 años $(\bar{X}=28, D E=8.3)$, el $83 \%$ refirió ser soltero, más de la mitad de los participantes cursó una licenciatura (53.8\%), seguido por preparatoria/bachillerato $(21.7 \%)$, carrera técnica $(10.4 \%)$, maestría $(6.6 \%)$, secundaria $(4.7 \%)$ y doctorado (2.8\%).

Respecto a los datos sobre historia sexual del participante, el $58.5 \%$ se identificó como bisexual, el $28.3 \%$ como homosexual/gay y el $13.2 \%$ como heterosexual. La media de la edad en que tuvieron la primera relación sexual fue de 17 años $(D E=3.5)$, la edad mínima de 12 y la máxima de 27años; el número de parejas sexuales en el último año osciló entre 2 y 30 con una media de 6.7 parejas ( $D E=6.68)$. La media de edad de uso de condón por primera vez fue de 18 años $(D E=3.7)$; más de la mitad de los participantes usó condón en la primera relación sexual (53.8\%) y en la última relación sexual (64.2\%). Así mismo, el 77.4\% refirió que su última relación sexual fue con un hombre; el $18.9 \%$ ha pagado por sexo y el $21.7 \%$ ha recibido alguna remuneración a cambio de este. En cuanto al conocimiento del estado serológico, el $72.6 \%$ refirió ser VIH negativo y el resto lo desconoce. (27.4\%). El 71.7\% se realiza la prueba de detección del $\mathrm{VIH}$ al menos una vez al año y el resto nunca se la ha realizado.

En la Tabla 1 se observa que los ítems para medir hipermasculinidad y uso de condón, se ubicaron por debajo del punto intermedio, lo que significa que el $50 \%$ de los participantes de la muestra (percentil 50), presentan puntuaciones de $\leq 22(\bar{X}=22.49, D E=6.8) ;(\bar{X}=21.94, D E=$ 4.78), respectivamente.

Tabla 1

Medidas de tendencia central y dispersión de las variables del estudio

\begin{tabular}{lcccccc}
\hline Variable & Media & Mdn & DE & Rango & Mínimo & Máximo \\
\hline Hipermasculinidad & 22.49 & 22 & 6.8 & 25 & 13 & 38 \\
Uso del condón & 21.94 & 22 & 4.78 & 21 & 11 & 32 \\
\hline
\end{tabular}

Nota: $\mathrm{DE}=$ Desviación estándar; $\mathrm{Mdn}=$ Mediana. $\mathrm{N}=106$.

En la Tabla 2 se pueden observar los porcentajes obtenidos de cada uno de los ítems que componen la subescala de ideología hipermasculina, al respecto, los porcentajes más altos se sitúan en las opciones de respuesta "totalmente en desacuerdo" y "en desacuerdo". Específicamente, el 59.4\% los participantes, están totalmente en desacuerdo en que los hombres no deben mostrar su afecto con los demás y que no deberían tener amigos homosexuales. Así mismo, el 25.5\% indicó que, para cuidar de su familia, es necesario tener el trabajo mejor pagado y el $29.2 \%$ mostró que, para un hombre, ser atlético o bueno en algún deporte es importante. 
Tabla 2

Hipermasculinidad

\begin{tabular}{|c|c|c|c|c|}
\hline Ítems & $\begin{array}{c}\text { Totalmente en } \\
\text { desacuerdo }\end{array}$ & $\begin{array}{c}\text { En } \\
\text { desacuerdo }\end{array}$ & $\begin{array}{c}\text { De } \\
\text { acuerdo }\end{array}$ & $\begin{array}{l}\text { Totalmente } \\
\text { de acuerdo }\end{array}$ \\
\hline & $\%$ & $\%$ & $\%$ & $\%$ \\
\hline
\end{tabular}

1. Un hombre debe probar su masculinidad teniendo relaciones sexuales con muchas personas.

2. Un hombre debe tener relaciones sexuales tan pronto como pueda en su vida.

3. Los hombres no deben llorar incluso cuando algo realmente malo sucede.

4. En una relación de pareja, los hombres deben tener relaciones sexuales con la mayor frecuencia posible

5. Un hombre siempre debe tener una mujer con la que esté saliendo.

6. Para ser un hombre, tienes que ser duro.

7. Si un hombre no es rico, debería intentar lucir como tal.

8. Un hombre no debe mostrar su afecto con los que ama

9. Un hombre no debería tener amigos varones que sean homosexuales

10. La mejor manera en que un hombre puede cuidar de su familia es conseguir el trabajo mejor pagado.

11. La ira es una emoción natural que un hombre debe demostrar.

12. Ser atlético o bueno en un deporte debe ser importante para un hombre.

13. Los hombres no deben tratar de resolver los problemas peleando.

$\begin{array}{llllllll}51 & 48.1 & 42 & 39.6 & 12 & 11.3 & 1 & 0.9\end{array}$

$\begin{array}{llllll}45 & 42.5 & 51 & 48.1 & 10 & 9.4\end{array}$

$\begin{array}{llllll}59 & 55.7 & 41 & 38.7 & 6 & 5.7\end{array}$

$\begin{array}{llllll}38 & 35.8 & 54 & 50.9 & 14 & 13.2\end{array}$

$\begin{array}{llllll}51 & 48.1 & 34 & 32.1 & 21 & 19.8\end{array}$

$\begin{array}{llllllll}55 & 51.9 & 40 & 37.7 & 10 & 9.4 & 1 & 0.9\end{array}$

$\begin{array}{llllllll}55 & 51.9 & 42 & 39.6 & 9 & 8.5 & -\end{array}$

$\begin{array}{llllllll}63 & 59.4 & 35 & 33.0 & 6 & 5.7 & 2 & 1.9\end{array}$

$\begin{array}{llllllll}63 & 59.4 & 36 & 34.0 & 6 & 5.7 & 1 & 0.9\end{array}$

$\begin{array}{llllllll}31 & 29.2 & 39 & 36.8 & 27 & 25.5 & 9 & 8.5\end{array}$

$\begin{array}{llllllll}47 & 44.3 & 51 & 48.1 & 7 & 6.6 & 1 & 0.9\end{array}$

$\begin{array}{llllllll}37 & 34.9 & 32 & 30.2 & 31 & 29.2 & 6 & 5.7\end{array}$

$\begin{array}{llllllll}19 & 17.9 & 20 & 18.9 & 31 & 29.2 & 36 & 34.0\end{array}$

Nota: $\mathrm{N}=106$. 
En cuanto al uso de condón, se observa que el 34.9\% siempre lleva un condón cuando saben que existe la posibilidad de tener relaciones sexuales con otra persona, el 31.1\% indicó que siempre insiste para usar el condón en las relaciones sexuales, sin embargo, el 29.2\% siempre tiene sexo oral sin usar condón. Además, el $49.1 \%$ algunas veces tuvo sexo anal sin usar condón, el $40.6 \%$ algunas veces tiene un plan en mente para practicar sexo seguro y el $39.6 \%$ algunas veces se niega a tener relaciones sexuales si su pareja insiste en tener sexo sin condón (Tabla 3 ).

Tabla 3

Uso de condón

\begin{tabular}{|c|c|c|c|c|c|c|c|c|}
\hline \multirow[t]{2}{*}{ Ítems } & \multicolumn{2}{|c|}{ Nunca } & \multicolumn{2}{|c|}{$\begin{array}{l}\text { Algunas } \\
\text { veces }\end{array}$} & \multicolumn{2}{|c|}{$\begin{array}{l}\text { La mayor } \\
\text { parte de } \\
\text { las veces }\end{array}$} & \multicolumn{2}{|c|}{ Siempre } \\
\hline & $f$ & $\%$ & $f$ & $\%$ & $f$ & $\%$ & $f$ & $\%$ \\
\hline $\begin{array}{l}\text { 1.Yo insisto con el uso del } \\
\text { preservativo cuanto tengo } \\
\text { relaciones sexuales. }\end{array}$ & 9 & 8.5 & 38 & 35.5 & 26 & 24.5 & 33 & 31.1 \\
\hline $\begin{array}{l}\text { 2. Yo detengo el juego previo a la } \\
\text { relación sexual (como } \\
\text { tocar/besar/agasajar) a tiempo } \\
\text { para ponerme un condón o para } \\
\text { que mi pareja se lo ponga. }\end{array}$ & 7 & 6.6 & 41 & 38.7 & 28 & 26.4 & 30 & 28.3 \\
\hline $\begin{array}{l}\text { 3. Sí sé que en un encuentro con } \\
\text { alguien podemos tener relaciones } \\
\text { sexuales, llevo un condón } \\
\text { conmigo. }\end{array}$ & 2 & 1.9 & 34 & 32.1 & 33 & 31.1 & 37 & 34.9 \\
\hline $\begin{array}{l}\text { 4. Si me dejo llevar por la pasión } \\
\text { del momento, yo tengo relaciones } \\
\text { sexuales sin usar un condón. }\end{array}$ & 25 & 23.6 & 50 & 47.2 & 25 & 23.6 & 6 & 5.7 \\
\hline $\begin{array}{l}\text { 5. Tengo sexo oral sin usar barrera } \\
\text { de protección, como un condón o } \\
\text { barrera de látex. }\end{array}$ & 12 & 11.3 & 36 & 34.0 & 27 & 25.5 & 31 & 29.2 \\
\hline $\begin{array}{l}\text { 6. Si sé que en un encuentro con } \\
\text { alguien podemos tener relaciones } \\
\text { sexuales, yo tengo un plan en } \\
\text { mente para practicar el sexo } \\
\text { seguro. }\end{array}$ & 5 & 4.7 & 43 & 40.6 & 29 & 27.4 & 29 & 27.4 \\
\hline $\begin{array}{l}\text { 7. Si mi pareja insiste en tener } \\
\text { relaciones sexuales sin usar } \\
\text { condón, me niego a tener } \\
\text { relaciones sexuales. }\end{array}$ & 25 & 23.6 & 42 & 39.6 & 21 & 19.8 & 18 & 17.0 \\
\hline $\begin{array}{l}\text { 8. Tengo relaciones sexuales } \\
\text { anales sin usar condón. }\end{array}$ & 38 & 35.8 & 52 & 49.1 & 12 & 11.3 & 4 & 3.8 \\
\hline
\end{tabular}

Nota: $\mathrm{N}=106$. 
Respecto al análisis correlacional, se encontró que la hipermasculinidad está relacionada de manera significativa y negativa con el uso de condón $\left(r_{s}=-.326, p=.001\right)$. En el análisis de regresión lineal, se consideró el uso de condón como variable dependiente y como independiente la hipermasculinidad, se observó que el modelo mejora significativamente la predicción de la variable dependiente $\left(F_{[1,104]}=11.676, p=.001\right)$, y la hipermasculinidad explica el $10 \%$ de la varianza del uso de condón. Después de ajustar el modelo (Tabla 4) se encontró que la hipermasculinidad influye de manera negativa sobre el uso de condón ( $b=-.318, p=.001$ ).

Tabla 4

Modelo de regresión lineal

\begin{tabular}{lllllll}
\hline & \multirow{2}{*}{$B$} & \multirow{2}{*}{$*$} & $p$ & \multicolumn{2}{c}{ IC 95\% } \\
\cline { 5 - 7 } & & & & $\mathrm{LI}$ & $\mathrm{LS}$ \\
\hline Hipermasculinidad & -.318 & -3.417 & .001 & & -.353 & -.094 \\
\hline
\end{tabular}

Nota: LI= Límite inferior; LS= Límite superior.

\section{Discusión}

Se observó que la mitad de los participantes HSHM presentaron puntuaciones por debajo del punto intermedio respecto a la subescala de hipermasculinidad, este hallazgo es similar a los resultados encontrados en un estudio realizado en esta población por LaPollo, Bond y Lauby (2014), quienes identificaron que la media de hipermasculinidad puntuaron por debajo del punto intermedio. En este sentido, la similitud de los puntajes puede explicarse por el uso del mismo instrumento de medición y la semejanza de las muestras. Esto podría indicar que este grupo específico de HSHM tiene una ideología alejada del modelo hegemónico, es importante considerar que en este estudio se observó una heterogeneidad en la orientación sexual y solo una pequeña parte se identificó como heterosexual, lo que demuestra que probablemente caen en el heterosexismo homofóbico, que propone como sujeto ideal al que realiza prácticas heterosexuales y rechaza las homosexuales, especialmente aquellas en la que se puede estar en posición pasiva (Bonino, 2003). En este sentido, los hombres con conductas no heterosexuales tienden a presentar menos ideas hipermasculinas, en comparación con los hombres exclusivamente heterosexuales.

Así mismo, los resultados obtenidos difieren con lo encontrado por Daniel y Bridges (2013), quienes muestran puntajes por encima de la media. La discordancia entre estos resultados podría explicarse, debido a que la construcción de la ideología masculina puede variar entre las diferentes sociedades (Connell, 1995; Courtenay, 2000; Gilmore, 1990). Al respecto, West y Zimmerman (1987) identificaron que la ideología de la masculinidad no solo se da por los comportamientos de los hombres, sino también a través de las dinámicas e interacciones sociales que se encargan de interpretar y valorar esos comportamientos. Además, el instrumento data de 1998, más de 20 años en donde el movimiento feminista en busca de la igualdad, además de las políticas públicas que promueven la incorporación de los varones en el cuidado de la salud sexual, pueden haber permeado de alguna forma en los varones "provocando cambios" en las formas de actuar y de sentir, o negociar el uso de condón y eso queda claro en las respuestas que dieron al instrumento de hipermasculinidad. La masculinidad en las últimas décadas de ha ido deconstruyendo, aunque aún se ven algunas resistencias al cambio. 
Se encontró que la hipermasculinidad se relaciona con el uso de condón. Estos resultados coinciden con Berg (2009), quien encontró que la masculinidad es un factor asociado con la práctica "barebacker", la cual consiste en no usar condones durante las relaciones sexuales de manera intencional. También, es consistente con lo demostrado por Dowsett, Williams, Ventuneac y Carballo-Diéguez, (2008), Halkitis y Parsons, (2003), Halkitis, Parsons y Wilton, (2003), Ridge, (2004), quienes reportaron que los HSH informan que no usar condones, los hace ser más hombres. Además, Hamilton y Mahalik (2009) encontraron que la ideología de masculinidad estaba correlacionada con la frecuencia de las relaciones sexuales sin protección y con la creencia de que el sexo sin protección es algo normativo. Se puede justificar la investigación para explorar más a fondo las intersecciones entre estos factores y cómo pueden influir en el riesgo de VIH entre los HSHM.

El análisis de regresión lineal reveló que las ideas hipermasculinas están asociadas a un menor uso de condón. Al respecto, la literatura muestra que los hombres con ideas hipermasculinas tienen malas actitudes hacia el uso de condón y también son quienes toman la decisión de usarlo o no durante un encuentro sexual (Ajzen, 2012). Además, otros factores que pueden afectar la decisión de usar condón son el deseo sexual, la búsqueda de sensaciones sexuales, la situación del momento y no tenerlo disponible al momento del acto. La agrupación de estos factores con la masculinidad hegemónica, disminuyen la posibilidad de usar condones en encuentros sexuales, por lo que representa un riesgo de adquirir el VIH (Marques, Junior, Gomes, \& Nascimento, 2012). El estudio presentó algunas limitaciones que se deben tomar en cuenta para futuras investigaciones. Dado que es un estudio transversal, no se puede establecer una direccionalidad en las asociaciones observadas y no permite determinar relaciones causales entre las variables; además, es importante mencionar que los resultados del estudio sólo son representativos de HSHM que tienen acceso a internet. Por lo tanto, las conclusiones obtenidas pueden ser sólo aproximaciones.

\section{Conclusiones}

Las ideas hipermasculinas, están asociadas a menor uso de condón en los HSHM. En este sentido, es importante que los profesionales de la salud exploren la ideología de masculinidad con la finalidad de identificar los componentes positivos que podrían ayudar en la realización de conductas sexuales seguras. Los hallazgos sugieren que existe la necesidad de diseñar intervenciones específicas en relación con la hipermasculinidad en el contexto del grupo de HSHM enfocadas a aumentar el uso de condón. Esto puede contribuir a la disminución de infecciones de transmisión sexual, entre ellas, el riesgo de adquirir el VIH.

\section{Referencias}

Ajzen, I. (2012a). Attitudes and persuasion. In K. Deaux \& M. Snyder (Eds.), The Oxford Handbook of Personality and Social Psychology (pp. 367-393). New York: Oxford University Press.

Baltar, F., \& Gorjup, M. T. (2012). Muestreo mixto online: Una aplicación en poblaciones ocultas. Intangible Capital, 8(1), 123-149. doi: http://dx.doi.org/10.3926/ic.294

Bianchi, M., Piccoli, V., Zotti, D., Fasoli, F., \& Carnaghi, A. (2017). The impact of homophobic labels on the internalized homophobia and body image of gay men: The moderation role of coming-out. Journal of Language and Social Psychology, 36(3), 356-367. doi: https://doi.org/10.1177/0261927X16654735. 
Bonino, L. (2003). Masculinidad hegemónica e identidad masculina. Dossiers feministes,6, 7-36. https://www.raco.cat/index.php/DossiersFeministes/article/view/102434

Burns, N., \& Grove, S. K. (2012). Cap. Población y muestras de investigación. Investigación en Enfermería, 5a Edición, Elsevier Saunders. Pp. 296-333.

Centro Nacional para la Prevención y Control del VIH y el sida [CENSIDA]. (2019). La epidemia del VIH y el sida en México. Hoja informativa. Recuperado de: http://www.censida.salud.gob.mx/descargas/epidemiologia/L_E V S.pdf

Centers for Disease Control and Prevention. (2017). Fact sheet: HIV/AIDS among African Americans. Atlanta, GA: Author.

Clark, J., Salvatierra, J., Segura, E. et al. (2013). Modern Love: Sexual Role-Based Identities and HIV/STI Prevention Among Men Who Have Sex with Men in Lima, Peru. AIDS Behavior 17, 1313-1328. doi:https://doi.org/10.1007/s10461-012-0210-5

Connell, RW (1995). Masculinities. Berkeley: University of California Press

Courtenay. W. H. (2000). Constructions of masculinity and their influence on men's well-being: a theory of gender and health. Social science \& medicine, 50(10), 1385-1401. doi: https://doi.org/10.1016/50277-9536(99)00390-1

Daniel S., \& Bridges, S. K. (2013). The relationships among body image, masculinity, and sexual satisfaction in men.Psychology of men \& masculinity,14(4), 345.doi: https://psycnet.apa.org/doi/10.1037/a0029154

da Fonte, Vinícius Rodrigues Fernandes da, Pinheiro, Carina D’Onofrio Prince, Barcelos, Nathália de Souza, Costa, Cristiane Maria Amorim, Ribeiro, Francisco Marcio Tadeu, \& Spindola, Thelma. (2017). Factores asociados con el uso del preservativo entre hombres jóvenes que tienen sexo con hombres. Enfermería Global,16(46), 50-93. doi: https://dx.doi.org/10.6018/eglobal.16.2.245451

Dilorio, C., Parsons, M., Lehr, S., Adame, D., \& Carlone, J. (1992). Measurement of Safe Sex Behavior In Adolescents and Young Adults. Nursing Research, 41(4), 203-209. doi:10.1097/00006199-199207000-00003

Doss, B. D., \& Hopkins, J. R. (1998). The multicultural masculinity ideology scale: Validation from three cultural perspectives. Sex Roles, 38, 719-741. doi: https://doi.org/10.1023/A:1018816929544

Dowsett, G., Williams, H., Carballo-Diéguez, A., \& Ventuneac, A. (2008). "Taking it like a man" Masculinity and barebacking online. Sexualities, 11(1),121-141. doi: $\underline{10.1177 / 1363460707085467}$

Ellen, J. M., Greenberg, L., Willard, N., Stines, S., Korelitz, J., \& Boyer, C. B. (2015). Cross-sectional survey comparing HIV risk behaviours of adolescent and young adult men who have sex with men only and men who have sex with men and women in the US and Puerto Rico.Sexually Transmitted Infections, 91(6), 458-461. doi: http://dx.doi.org/10.1136/sextrans-2014-051712 
Friedman, M. R., Stall, R., Silvestre, A. J., Mustanski, B., Shoptaw, S., Surkan, P. J., \& Plankey, M. W. (2014). Stuck in the middle: longitudinal HIV-related health disparities among men who have sex with men and women (MSMW). Journal of acquired immune deficiency syndromes (1999),66(2), 213. https://dx.doi.org/10.1097\%2FQAl.0000000000000143

Gilmore, D. D. (1990). Manhood in the making: Cultural concepts of masculinity. Yale University Press.

Grov, C., Golub, S. A., Mustanski, B., \& Parsons, J. T. (2010). Sexual compulsivity, state affect, and sexual risk behavior in a daily diary study of gay and bisexual men. Psychology of Addictive Behaviors, 24(3), 487. doi: https://psycnet.apa.org/doi/10.1037/a0020527

Gutiérrez, J. P., Sucilla-Pérez, H., Conde-González, C. J., Izazola, J. A., Romero-Martínez, M., \& Hernández-Ávila, M. (2014). Seroprevalencia de VIH en población mexicana de entre 15 y 49 años: resultados de la Ensanut 2012. Salud Pública de México, 56(4), 323-332 doi: https://doi.org/10.21149/spm.v56i4.7352

Halkitis, P. N., \& Parsons, J. T. (2003). Intentional unsafe sex (barebacking) among HIV-positive gay men who seek sexual partners on the Internet. AIDS Care,15(3), 367-378. doi: https://doi.org/10.1080/0954012031000105423

Halkitis, P.N., Parsons, J.T. \& Wilton, L. (2003). Barebacking Among Gay and Bisexual Men in New York City: Explanations for the Emergence of Intentional Unsafe Behavior. Arch Sex Behavior 32, 351-357. doi: https://doi.org/10.1023/A:1024095016181

Hamilton, C. J., \& Mahalik, J. R. (2009). Minority stress, masculinity, and social norms predicting gay men's health risk behaviors. Journal of Counseling Psychology, 56(1), 132-141. doi: https://doi.org/10.1037/a0014440

Jiménez V. V. (2018). Modelo de conducta sexual segura en hombres que tienen sexo con hombres (Tesis de Doctorado). Universidad Autónoma de Nuevo León.

Knox, J., Reddy, V., Kaighobadi, F, Nel, D., \& Standford, T. (2013). Communicating HIV Status in Sexual Interactions: Assessing Social Cognitive Constructs, Situational Factors, and Individual Characteristics Among South African MSM. AIDS Behavior,17, 350-359. doi: https://doi.org/10.1007/s10461-012-0337-4

LaPollo, A. B., Bond, L., \& Lauby, J. L. (2014). Hypermasculinity and sexual risk among Black and White men who have sex with men and women. American journal of men's health, 8(5), 362-372. doi: https://doi.org/10.1177\%2F1557988313512861

Marques, J.S., Junior, Gomes, R., \& Nascimento, E.F. (2012). Masculinidade hegemônica, vulnerabilidade e prevenção ao HIV/AIDS. Ciênc Saúde Coletiva,17(2):511-20. doi: http://dx.doi.org/10.1590/S1413-81232012000200024

Mahalik, J.R., Burns, S.M. and Syzdek, M. (2007) Masculinity and Perceived Normative Health Behaviors as Predictors of Men's Health Behaviors. Social Science \& Medicine, 64, 22012209. doi: http://dx.doi.org/10.1016/i.socscimed.2007.02.035 
Martínez-Torres, Javier, Parada-Capacho, Lince Yurley, \& Castro-Duarte, Zolly Klarenna. (2014). Prevalencia de uso de condón en todas las relaciones sexuales con penetración durante los últimos doce meses y factores asociados en estudiantes universitarios entre 18 y 26 años de edad. Universidad y Salud, 16(2), 198-206. Recuperado November 03, 2019, from http://www.scielo.org.co/scielo.php?script=sci_arttext\&pid=S01247107201400020000 $7 \& \operatorname{lng}=$ en\&tng=

Maulsby, C., Sifakis, F., German, D., Flynn, C. P., \& Holtgrave, D. (2013). HIV risk among men who have sex with men only (MSMO) and men who have sex with men and women (MSMW) in Baltimore. Journal of homosexuality, 60(1), 51-68. doi: https://doi.org/10.1080/00918369.2013.735938

McCree, D. H., Oster, A. M., Jeffries IV, W. L., Denson, D. J., Lima, A. C., Whitman, H., \& Henny, K. D. (2017). HIV acquisition and transmission among men who have sex with men and women: what we know and how to prevent it. Preventive medicine, 100, 132-134. doi: https://doi.org/10.1016/j.ypmed.2017.04.024

O'Neil, J.M., Helms, B.J., Gable, R.K. et al. (1986). Gender-role conflict scale: College men's fear of femininity. Sex Roles 14, 335-350. doi: https://doi.org/10.1007/BF00287583

Oparanozie, A., Sales, J. M., DiClemente, R. J., \& Braxton, N. (2012). Racial identity and risky sexual behaviors among black heterosexual men. Journal of Black Psychology, 38, 38-51.doi: https://doi.org/10.1177/0095798410397542

Programa Conjunto de las Naciones Unidas dedicado al VIH/Sida [ONUSIDA]. (2017). Estadísticas de América Latina sobre el VIH. Hoja informativa, Julio 2017. Recuperado de: http://onusidalac.org/1/images/america-latina-datos-2017.pdf

Rhodes, S. D., Hergenrather, K. C., Vissman, A. T., Stowers, J., Davis, A. B., Hannah, A., . . Marsiglia, F. F. (2011). Boys must be men, and men must have sex with women: A qualitative CBPR study to explore sexual risk among African American, Latino, and white gay men and MSM. American Journal of Men's Health, 5, 140-151. doi: https://doi.org/10.1177/1557988310366298

Ridge, D. T. (2004). "It was an incredible thrill": The social meanings and dynamics of younger gay men's experiences of barebacking in Melbourne. Sexualities, 7, 259-279. doi: https://doi.org/10.1177/1363460704040138

Santana, M.C., Raj, A., Decker, M.R. et al. (2006). Masculine Gender Roles Associated with Increased Sexual Risk and Intimate Partner Violence Perpetration among Young Adult Men. Journal of Urban Health 83, 575-585 doi: https://doi.org/10.1007/s11524-006$\underline{9061-6}$

Scanavino, M., Ventuneac, A., Abdo, C., Tavares, H., Amaral, M., Messina, B., Reis, S. C., Martins, J., \& Parsons, J. T. (2018). Sexual compulsivity, anxiety, depression, and sexual risk behavior among treatment-seeking men in São Paulo, Brazil. Revista brasileira de psiquiatria (Sao Paulo, Brazil : 1999), 40(4), 424-431. doi: https://doi.org/10.1590/1516$\underline{4446-2017-2476}$ 
Shadaker, S., Magee, M., Paz-Bailey, G., Hoots, B. E., \& NHBS Study Group. (2017). Characteristics and risk behaviors of men who have sex with men and women compared with men who have sex with men-20 US cities, 2011 and 2014. Journal of Acquired Immune Deficiency Syndromes, 75, S281-S287. doi: http://10.1097/QAl.0000000000001403

Valdez, M. C. (2015). Modelo de Conducta sexual en hombres que tienen sexo con hombres (Tesis de doctorado). Universidad Autónoma de Nuevo León.

Viveros, V., M. (2001). Contemporary Latin American Perspectives on Masculinity. Men and Masculinities, 3 (3), 237-260. doi: https://doi.org/10.1177/1097184X01003003002

West, C., \& Zimmerman, D. H. (1987). Doing gender. Gender \& Society, 1(2), 125-151.doi: https://doi.org/10.1177\%2F0891243287001002002

Xu, W., Zheng, L., Liu, Y., \& Zheng, Y. (2016). Sexual sensation seeking, sexual compulsivity, and high-risk sexual behaviours among gay/bisexual men in Southwest China. AIDS care, 28(9), 1138-1144. doi: https://doi.org/10.1080/09540121.2016.1153587

Zeglin, R. J., \& Stein, J. P. (2015). Social determinants of health predict state incidence of HIV and AIDS: A short report. AIDS care, 27(2), 255-259. doi: https://doi.org/10.1080/09540121.2014.954983 